\title{
Produção do conhecimento sobre promoção da saúde e prática da enfermeira na Atenção Primária
}

\author{
Production of knowledge on health promotion and nurse's practice in Primary Health Care \\ Producción de conocimiento acerca de la promoción de la salud y práctica de la enfermera \\ en la Atención Primaria
}

\section{Nildo Batista Mascarenhas', Cristina Maria Meira de Melo", Norma Carapiá Fagundes" \\ ' Universidade Federal da Bahia, Escola de Enfermagem, Programa de Pós-Graduação em Enfermagem (Mestrando). Salvador-BA, Brasil. \\ "Universidade Federal da Bahia, Escola de Enfermagem, Departamento de Enfermagem Comunitária, Programa de Pós-Graduação em Enfermagem. Salvador-BA, Brasil.}

\section{Submissão: 08-09-2011 Aprovação: 26-11-2012}

\section{RESUMO}

Trata-se de uma revisão integrativa que analisa a produção científica nacional sobre a promoção da saúde e a prática da enfermeira no campo da atenção primária. Com o desenvolvimento do estudo, verificou-se que a discussão deste tema é pouco explorada no campo da Enfermagem e que a promoção da saúde ainda é compreendida como sinônimo da educação em saúde e/ou da prevenção de doenças. Conclui-se que a educação em saúde é uma estratégia essencial para o alcance da promoção da saúde e que uma não se resume à outra. Além disso, é necessário que as enfermeiras reconheçam a promoção da saúde como um campo de práxis capaz de transformar a realidade social e sua própria prática.

Descritores: Promoção da Saúde; Atenção Primária; Educação em Saúde; Enfermagem.

\section{ABSTRACT}

This is an integrative review that examines the national scientific production on health promotion and nurse's practice in the field of primary health care. With the development of the study, it was found that the discussion of this theme is little explored in nursing and that health promotion is still understood as synonymous of health education and / or prevention of disease. It is concluded that health education is an essential strategy to achieve the health promotion, understanding that one is not restricted to the other. Moreover, it is necessary that nurses recognize health promotion as a field of practice can transform social reality and their own practice.

Key words: Health Promotion; Primary Health Care; Health Education; Nursing.

\section{RESUMEN}

Esta es revisión integradora que analiza la producción científica nacional acerca de la promoción de la salud y práctica de la enfermera en la atención primaria. Con el desarrollo del estudio, se encontró que la discusión de este tema es poco explorada en la enfermería y que y la promoción de la salud sigue siendo entendida como sinónimo de educación para la salud y / o de prevención de enfermedades. Se concluye que la educación para la salud es una estrategia esencial para el logro de promoción de la salud, con el entendimiento de que una no se limita a otra. También es necesario que las enfermeras reconocen la promoción de la salud como un campo de praxis que puede transformar la realidad social y su propia práctica.

Palabras clave: Promoción de la Salud; Atención Primaria; Educación para la Salud; Enfermería.

Extraído da monografia "Promoção da Saúde e a Prática do Enfermeiro na Atenção Primária: contribuição ao estudo", apresentada, em 2010, à Escola de Enfermagem da UFBA. Integra a pesquisa "Análise do trabalho e do processo de educação permanente da enfermeira no contexto do SUS", que conta com apoio financeiro da Fundação de Amparo à Pesquisa do Estado da Bahia. 


\section{INTRODUÇÃO}

A promoção da saúde, considerada atualmente um campo conceitual e de práxis, tem influenciado a organização do sistema de saúde de diversos países e regiões do mundo ${ }^{(1)} \mathrm{Em}$ sua conformação teórica moderna, a promoção da saúde é a intervenção sobre as condições de vida da população; extrapola a prestação de serviços clínico-assistenciais e preconiza ações intersetoriais que envolvem a educação, o saneamento básico, a habitação, a renda, o trabalho, a alimentação, o meio ambiente, o acesso a bens e serviços essenciais, o lazer, dentre outros determinantes sócio-ambientais que incidem na produção da saúde e da doença ${ }^{(2)}$.

Promover a saúde constitui um desafio, pois sua abrangência de ação é maior que o campo específico da saúde ${ }^{(3)}$ e envolve uma combinação de estratégias, a saber: ações do Estado (políticas públicas saudáveis), da comunidade (reforço da ação comunitária), de indivíduos (desenvolvimento de habilidades pessoais), do sistema de saúde (reorientação do sistema de saúde) e de parcerias intersetoriais ${ }^{(4)}$. Para isso, é essencial a responsabilidade conjunta dos sujeitos implicados no processo saúde-doença-cuidado, seja na identificação dos problemas, seja nas soluções propostas para os mesmos, o que demanda uma ação coordenada entre todas as partes envolvidas ${ }^{(4)}$

Assim, ao partir de uma perspectiva contra-hegemônica do modelo assistencial e de organização dos sistemas de saúde, considera-se que a promoção da saúde configura-se como um movimento ideológico.

A ideologia pode ser compreendida como o sistema de idéias e representações que dominam o espírito de uma pessoa ou de um grupo social e a prática ideológica compreende as atividades realizadas no sentido de transformar uma dada ideologia em outra(5). Nesse sentido, “(...) um movimento ideológico representa um conjunto de atividades visando transformar a 'visão de mundo' dos homens em uma nova "visão de mundo"(5).

O movimento da promoção da saúde vislumbra mais que a produção de novos conhecimentos e mudanças na estrutura da atenção à saúde. Enquanto movimento ideológico, a promoção da saúde construiu diversas críticas às práticas hegemônicas de saúde e propõe uma mudança baseada na transformação da atenção à saúde, com projeções para a organização social em que se vive" ${ }^{\prime \prime(5)}$.

Historicamente, a promoção da saúde desponta internacionalmente como uma nova concepção de saúde em meados da década de 1970, resultado do debate, na década anterior, sobre a determinação social e econômica da saúde ${ }^{(1)}$ Embora o termo tenha sido utilizado nessa mesma década pelos sanitaristas Hugh Rodney Leavell e Edwin Gurney Clark para caracterizar um nível de atenção da medicina preventiva, seu significado foi mudando, passando a representar, mais recentemente, um enfoque político e técnico em torno do processo saúde-doença-cuidado(4).

No campo das definições, verifica-se que o conceito contemporâneo de promoção da saúde é multifacetado e ainda está em construção. Comumente, o campo de ação e a ideologia da promoção da saúde ainda são vinculados hegemonicamente às intervenções de educação em saúde tradicionais, que visam mudanças de comportamento dos sujeitos e a atividades clínico-preventivas. Isso se dá especialmente por conta da marcante influência do modelo da "História Natural das Doenças", proposto por Leavell e Clark, no cotidiano dos profissionais que atuam na atenção primária. Nesse modelo,

(...) o homem é colocado com seus atributos em um ponto; não é o homem como ser histórico em sua relação com a natureza através do trabalho, em que esta passa também a ser histórica, não é o homem constituído pelo conjunto de suas relações sociais, enfim, não é o homem que fala, produz e vive, mas o conjunto de seus atributos que se transformam em fatores de morbidade ${ }^{(5)}$.

Nos últimos trinta anos, a promoção da saúde caracteriza-se como uma estratégia promissora para enfrentar os diversos problemas de saúde que afetam as populações humanas. Esse movimento, ao partir de uma concepção ampliada do processo saúde-doença-cuidado e de seus determinantes, propõe ainda a articulação dos saberes técnicos e dos saberes populares, além da mobilização de recursos institucionais e comunitários para o enfrentamento e resolução dos problemas de saúde ${ }^{(4)}$

Na Carta de Ottawa, a promoção da saúde é concebida como um processo de capacitação da comunidade para atuar na melhoria de sua qualidade de vida e saúde, incluindo maior participação no controle deste processo. Partindo dessa concepção, pode-se dizer que a promoção da saúde, ao conferir possibilidades e liberdade aos indivíduos e grupos, parte de uma concepção ampliada da saúde, a qual deve ser vista como um recurso para a vida, e não como objetivo de viver; como um conceito positivo que enfatiza os recursos sociais e pessoais, bem como as capacidades físicas dos sujeitos. Sendo assim, operar a promoção da saúde não é responsabilidade exclusiva do setor saúde. Alcançar a promoção da saúde significa, também, extrapolar um estilo de vida saudável e caminhar em direção a um bem-estar de toda a sociedade.

Contudo, para compreender a promoção da saúde como mudança de paradigma, é preciso distingui-la da prevenção de doenças, dado que no imaginário social ainda persiste uma confusão conceitual e paradigmática entre promoção e prevenção.

Em linhas gerais, o discurso preventivista focaliza os aspectos biológicos, identifica riscos e atua sobre eles. O seu campo de ação fundamenta-se no modelo da História Natural das Doenças, no conceito ecológico de saúde/doença e tem como base o conhecimento epidemiológico moderno, objetivando o controle da transmissão de doenças infecciosas e a redução do risco de doenças degenerativas ou outros agravos específicos ${ }^{(3)}$.

Com essas considerações depreende-se que as ações preventivas não consideram a dimensão histórico-social do processo saúde-doença-cuidado, além de não estimular, nas coletividades, processos de ampliação do poder e de tomada de decisão em relação às políticas de saúde, de modo que as pessoas estejam implicadas na luta pela melhoria das suas condições de vida e trabalho, com vistas ao enfrentamento de seus problemas e a valorização de suas potencialidades ${ }^{(6)}$.

Em contrapartida, a promoção da saúde é uma estratégia que remete à dimensão social, existencial e ética dos sujeitos; 
ao seu engajamento e comprometimento, envolvendo o fortalecimento da capacidade individual e coletiva para lidar com a multiplicidade dos determinantes da saúde para além de uma aplicação técnica e normativa.

Nessa concepção, compreende-se que não basta conhecer o funcionamento das doenças e encontrar mecanismos para seu controle. A promoção da saúde diz respeito ao fortalecimento da saúde por meio da construção da capacidade de escolha ${ }^{(3)}$; da mediação entre as pessoas e seu ambiente, combinando escolhas individuais com responsabilidade social pela saúde ${ }^{(4)}$.

Por conta da sua peculiaridade, a Atenção Primária à Saúde (APS), compreendida em sua dimensão abrangente, representa um espaço privilegiado para as ações de promoção da saúde no âmbito do sistema de serviços. Isso se dá principalmente porque a APS é essencial para a estruturação de sistemas de saúde, quando compreendida como uma estratégia que possibilita a intervenção sobre os determinantes sociais do processo saúde-doença-cuidado, que são inerentes a atenção à saúde da população(7).

A atenção primária à saúde pode ser definida como o nível de um sistema de serviços de saúde que possibilita a obtenção de respostas para todas as necessidades e problemas. Este nível do sistema de saúde produz serviços e ações de atenção à pessoa no decorrer do tempo e em todas as suas condições de saúde ou doença, excetuando-se as muito incomuns e raras, e engloba um conjunto de funções que, combinadas, são exclusivas deste nível. A abordagem inerente à atenção primária forma a base e determina o trabalho de todos os outros níveis do sistema de saúde, de modo a coordenar, racionalizar e direcionar o uso dos recursos, básicos ou especializados, para a promoção, proteção e melhoria da saúde ${ }^{(8)}$.

A atenção primária revela-se como uma tendência relativamente recente de inverter a priorização das ações de saúde de uma abordagem desintegrada, curativa e centrada no papel hegemônico do médico, para uma abordagem preventiva e promocional, integrada com outros níveis de atenção, e construída coletivamente com outros profissionais de saúde ${ }^{(9)}$, em prol do desenvolvimento humano, social e econômico das populações. Frente ao exposto, atuar na atenção primária na perspectiva da promoção da saúde.

(...) configura-se como uma possibilidade de responder a demandas sociais e exige reflexões que perpassam quatro eixos fundamentais: a concepção de saúde, a gestão do processo de trabalho e educação, a formação dos profissionais de saúde, a participação e o controle social. A conjugação dos elementos destes eixos deve direcionar as práticas em saúde, imprimindo a lógica do modelo tecnoassistencial em constante construção e reconstrução ${ }^{(10)}$.

As enfermeiras, ao atuarem no campo da atenção primária e em consonância com os pressupostos da promoção da saúde, devem pautar a sua prática em uma concepção ampliada de saúde, considerando os determinantes sócio-ambientais do processo saúde-doença-cuidado. Devem também estimular e promover a participação política da comunidade; devem atuar de modo a extrapolar os limites dos serviços de saúde, com vistas a adotar ações intersetoriais e criar ambientes favoráveis à saúde; e devem se engajar na luta pela consolidação de políticas públicas saudáveis ${ }^{(7)}$.

Apesar da importante incorporação dos pressupostos da promoção da saúde na prática das enfermeiras no campo da atenção primária, é fundamental sinalizar que as mudanças nas ações de saúde direcionadas à construção da promoção da saúde ainda são incipientes e têm pouca visibilidade no cenário das práticas em saúde, justificando a não percepção desta prática como estratégia para as mudanças no estado de saúde da população(10). Além disso, é importante reconhecer que a maior parte dos profissionais desconhece o significado da promoção da saúde, o que acentua a confusão conceitual entre promoção e prevenção ${ }^{(1)}$.

A Enfermagem, compreendida como um campo de prática social que se relaciona com a estrutura econômica, política e ideológica da sociedade brasileira, e cuja essência tem uma forte conotação de promoção global da saúde, é um espaço fundamental para a consolidação da promoção da saúde no contexto da atenção primária. Sendo assim, a enfermeira, ao atuar na atenção primária, incorporando a promoção da saúde como uma estratégia de transformação social e política da saúde da comunidade, potencializa as ações desenvolvidas na APS, projetando a sua prática com mais autonomia em relação à prática médica, consoante com o conceito ampliado de saúde e com o cuidado integral, e articulando-se com os grupos da população onde atua para responder às necessidades de saúde da população e aos princípios do $\operatorname{SUS}^{(7)}$.

Na perspectiva da promoção da saúde, os cuidados em enfermagem têm por objetivo desenvolver a capacidade de indivíduos, famílias e comunidade para identificar as suas necessidades de saúde e participar, conjuntamente, na busca por soluções para elas, tendo em vista as possibilidades ao seu alcance ${ }^{(11)}$. Esses cuidados, sob a ótica da promoção da saúde, [...] exigem conhecimento das necessidades de saúde, a partir da descoberta das pessoas e do seu meio de vida, estabelecendo um laço entre a manifestação da necessidade e do problema de saúde e as condições de vida como a habitação, o trabalho, o transporte ${ }^{(12)}$.

Observa-se, assim, que os cuidados em enfermagem, desenvolvidos na perspectiva da promoção da saúde, são parte da atenção primária e constituem elemento fundamental para subsidiar a prática da promoção global da saúde nesse contexto. Esse fato deve-se principalmente à proximidade do cuidado em enfermagem com a promoção da vida.

As enfermeiras devem compreender que esse potencial é inerente à sua prática e à prática do cuidado. Isso nos leva a afirmar que o tratamento das doenças não deve ser a prática hegemônica da enfermeira, já que historicamente este é um constructo inerente à prática médica, e todas as ações desenvolvidas a partir dela são subsidiárias desta prática.

É preciso também enfatizar que a promoção da saúde é uma estratégia fundamental para impulsionar mudanças não somente no setor saúde, mas também na sociedade, resgatando o cuidado nas relações humanas e nas práticas de saúde e que, em conjunto com as premissas da atenção primária, possibilita o resgate de valores essenciais para a construção de novas relações sociais pautadas no respeito, na ética, na solidariedade, e no cuidado. 
Diante do exposto, e no intuito de suscitar reflexões e contribuir para o debate sobre a promoção da saúde na APS, objetiva-se analisar a produção científica nacional sobre promoção da saúde e prática da enfermeira no campo da atenção primária.

\section{MÉTODO}

Trata-se de uma revisão integrativa da literatura. Este método de pesquisa possibilita a síntese das informações disponíveis sobre determinado assunto, em um dado momento, podendo também apontar lacunas e inferir conclusões gerais a respeito de uma área particular de estudo ${ }^{(13)}$. Esse tipo de investigação disponibiliza um resumo das evidências mediante a aplicação de métodos explícitos e sistematizados de busca, apreciação crítica e síntese da informação selecionada ${ }^{(14)}$. Para operacionalizá-la, seguiram-se as seguintes etapas ${ }^{(13)}$ :

$\checkmark$ Formulação da questão de pesquisa;

$\checkmark$ Estabelecimento de critérios para inclusão de estudos na investigação;

$\checkmark$ Definição das informações a serem extraídas dos estudos selecionados/ categorização dos estudos;

$\checkmark$ Avaliação e análise crítica dos estudos incluídos na revisão;

$\checkmark$ Interpretação dos resultados;

$\checkmark$ Apresentação da conclusão/síntese do conhecimento.

Definiu-se a seguinte questão de pesquisa: qual a produção científica nacional sobre promoção da saúde e prática da enfermeira na atenção primária?

Os dados foram coletados no período de abril a junho de 2010, no site da Biblioteca Virtual em Saúde, utilizando-se as bases de dados Medical Literature Analysis and Retrieval System On-line (MEDLINE) e Literatura Latino-Americana e do Caribe em Ciências da Saúde (LILACS), além da coleção Scientific Electronic Library Online (SciELO ). Para a pesquisa nas bases de dados, utilizaram-se os seguintes descritores: atenção primária; enfermagem; enfermagem em saúde pública; prática profissional; programa saúde da família; promoção da saúde; trabalho.

Os critérios para inclusão dos artigos no estudo foram: que estivessem em português; que o texto completo estivesse disponível em suporte eletrônico; com dados coletados no Brasil; com o lócus de estudo na atenção primária e como sujeitos as enfermeiras.

A análise dos dados ocorreu por meio da leitura exploratória, seletiva e analítica, considerando as concepções ideológicas da promoção da saúde e da prática da enfermeira na atenção primária. Este processo ocorreu em três etapas ${ }^{(15)}$ : pré-análise, em que o material selecionado foi organizado, lido e as percepções registradas; exploração do material, que consistiu na transformação dos dados brutos, objetivando alcançar o núcleo de compreensão do texto, e análise final, que desvendou o conteúdo trabalhado, considerando as ideologias, tendências e outras denominações características do fenômeno em análise.

\section{PRODUÇÃO DO CONHECIMENTO SOBRE PROMOÇÃO DA SAÚDE E PRÁTICA DA ENFERMEIRA NA ATENÇÃO PRIMÁRIA}

Inicialmente, a busca realizada nas três bases de dados evidenciou um número considerável de material produzido, conforme explicitado na tabela 1.

Tabela 01 - Produção científica identificada nas bases de dados, relacionada ao tema em questão.

\begin{tabular}{cccc}
\hline Base de dados & $\begin{array}{c}\text { Total de produções } \\
\text { identificadas }\end{array}$ & $\begin{array}{c}\text { Produções em } \\
\text { texto completo }\end{array}$ & \% \\
\hline MEDLINE & 1829 & 94 & 5,1 \\
\hline LILACS & 314 & 129 & 41 \\
\hline SciELO & 36 & 36 & 100 \\
\hline Total & $\mathbf{2 1 7 9}$ & $\mathbf{2 5 9}$ & - \\
\hline
\end{tabular}

Dentre a produção disponível em texto completo, foram identificados cento e quarenta e nove (149) artigos em português, cem (100) em inglês, oito (8) em espanhol e dois (2) em francês. Deste total, e excluindo as repetições, foram selecionados dez artigos que atenderam aos critérios de inclusão, perfazendo um porcentual de $4 \%$ (Tabela 02).

O baixo porcentual de artigos demonstra que, apesar da promoção da saúde ser bastante enunciada por enfermeiras que atuam na atenção primária, a produção científica sobre a temática ainda é pouco explorada no campo da Enfermagem no Brasil.

Apesar da promoção da saúde ser discutida há mais de 30 anos, salienta-se que no Brasil as discussões desse campo ainda se encontram em difusão(16), o que pode justificar a pouca produção encontrada. Nesse contexto, com vistas à socialização do conhecimento e ao fortalecimento das ações no campo da promoção da saúde, a Política Nacional de Promoção da Saúde enfatiza a necessidade de articular ações intersetoriais e entre as esferas de governo para a difusão de experiências exitosas e de processos avaliativos nesse campo. Isso demonstra a importância para as enfermeiras produzirem conhecimento sobre a prática da promoção da saúde na atenção primária. Mas a ausência de produção de conhecimento neste campo pode significar que a promoção da saúde ainda não está incorporada à prática das enfermeiras brasileiras.

Quanto aos periódicos onde os artigos foram publicados, $80 \%$ da produção é concentrada naqueles diretamente relacionados à área de Enfermagem. Com relação às abordagens dos estudos, prevaleceram os relatos de experiência (50\%), seguidos pelos estudos empíricos (30\%), por uma revisão bibliográfica (10\%) e por um artigo de atualização (10\%). A análise dos objetivos dos artigos evidenciou um fato que merece ser discutido: $80 \%$ da produção (oito artigos) abordou a prática da enfermeira na educação em saúde. 
Tabela 02 - Artigos incluídos na revisão integrativa, segundo título, autor(es), ano, objeto de estudo, resultados e conclusões.

Título

Autor(es) Ano Objeto de estudo

Resultados

Conclusões

Práticas de educação em saúde: atividades de grupo voltadas para prevenção de doenças e promoção

Práticas educativas desenvolvidas por enfermeiros do

Programa Saúde da Família no Rio de Janeiro da saúde; capacitação dos agentes comunitários de saúde; visitas domiciliares; consulta individual e coletiva.

Heringer A, Ferreira VA, Acioli S, Barros ALS
Práticas educativas no Programa de desenvolvidas por enfermeiros Saúde da Família
Práticas de promoção da saúde: construção de grupos.

Dissociação entre o discurso e a prática: entrevistados referiram a utilização de práticas educativas diferenciadas. No entanto, na observação in locu, observou-se que estes utilizavam predominantemente uma prática educativa vertical.

Abordagens: identificou-se a educação em saúde tradicional e a não tradicional.
Dissociação entre o discurso e a prática.

Necessidade de articular teoria e prática.

Ainda que de modo sutil, tem ocorrido mudanças nas práticas e a incorporação de metodologias educativas transformadoras.

\begin{tabular}{|c|c|c|c|c|}
\hline $\begin{array}{l}\text { Programa de } \\
\text { prevenção e } \\
\text { tratamento do } \\
\text { tabagismo: } \\
\text { uma vivência } \\
\text { acadêmica de } \\
\text { enfermagem }\end{array}$ & $\begin{array}{l}\text { Machado } \\
\text { VC, Alerico } \\
\text { MI, Sena J }\end{array}$ & 2007 & $\begin{array}{l}\text { Vivência de } \\
\text { uma discente } \\
\text { de um curso de } \\
\text { graduação em } \\
\text { Enfermagem em } \\
\text { um Programa } \\
\text { de prevenção e } \\
\text { tratamento do } \\
\text { tabagismo. }\end{array}$ & $\begin{array}{l}\text { Algumas ações realizadas pelo } \\
\text { Programa de Prevenção e Tratamento } \\
\text { do Tabagismo: formação de grupos; } \\
\text { entrevista individual com cada } \\
\text { participante do Programa; discussão } \\
\text { sobre o que é tabaco. }\end{array}$ \\
\hline
\end{tabular}

Atividade educativa junto aos estudantes: palestra sobre doenças sexualmente transmissíveis e higiene. Os materiais didáticos utilizados foram: álbum seriado, prótese (pênis), preservativo masculino (camisinha), cartazes e quadro negro.
Os objetivos do referido Programa estão sendo alcançados, pois se percebeu que há pessoas que estão se causa males à saúde. conscientizando de que fumar
Educação em saúde: uma experiência transformadora

$\mathrm{HM}$, Gonçalves MJF. 2004 positiva transformadora da prática da educação em saúde
A educação em saúde é essencial para a reflexão e mudança de comportamento na vida dos indivíduos. Portanto, ela precisa ser sistematicamente planejada;

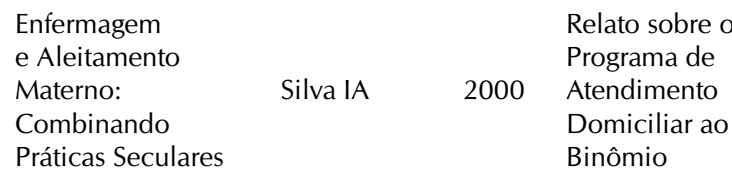

Ações realizadas: entrevistas e acompanhamento de gestantes; discussão sobre aspectos da amamentação; visitas domiciliares, em caso de intercorrências no processo de amamentação; orientação sobre técnicas de amamentação.
A experiência de atendimento domiciliar ao binômio mãefilho, durante a amamentação, é um campo com muitas possibilidades de atuação para os profissionais de Enfermagem.
Enfermagem em

Puericultura:

unindo

metodologias assistenciais para promover a saúde nutricional da criança
Silva MM,

Rocha L,

Silva SO
Experiência de estudantes de enfermagem em puericultura
Ações realizadas: reestruturação da assistência às crianças, utilizando para tanto visitas e consultas de enfermagem; ações educativas e assistenciais, visando promover a saúde de crianças.
A consulta de enfermagem, aliada à visita domiciliar, pode prevenir doenças e promover saúde. Além disso, quando associadas, pode-se construir ações educativas/assistenciais nas questões nutricionais e alimentares. 
Intersetorialidade: uma vivência prática ou um desafio a ser conquistado? O Discurso do Sujeito Coletivo dos enfermeiros nos núcleos de Saúde da Família do Distrito Oeste Ribeirão Preto
Paula KA Palha PF,

Protti ST
Compreensão

de enfermeiros

sobre a intersetorialidade
Os serviços de saúde e os sujeitos atuam de forma fragmentada.

O trabalho em equipe ainda é compartimentalizado.

A demanda impulsiona a busca pelas ações e contatos intersetoriais.

É um trabalho novo que está sendo construído e requer a formação.
A intersetorialidade é pouco compreendida e, por vezes, é confundida com interdisciplinaridade.

A intersetorialidade ocorre de forma informal.

É necessário incorporar a discussão sobre intersetorialidade na prática dos profissionais de saúde.

O grupo é um espaço de convívio com limitações, cumplicidade e identidade. estratégias utilizadas na Modos de cuidar em saúde pública: o trabalho grupal na rede básica de saúde

Abrahão

$\mathrm{AL}$, Freitas CSF
2008 construção e manutenção de um grupo educativo na atenção básica
As autoras teceram considerações sobre o conceito de grupo, o grupo no campo da saúde e na atenção básica, e sobre a coordenação de grupo.
Além disso, o grupo educativo pode estimular a criação de outras formas de lidar e conviver com a doença, e de um espaço de construção coletiva e singular de cuidar.

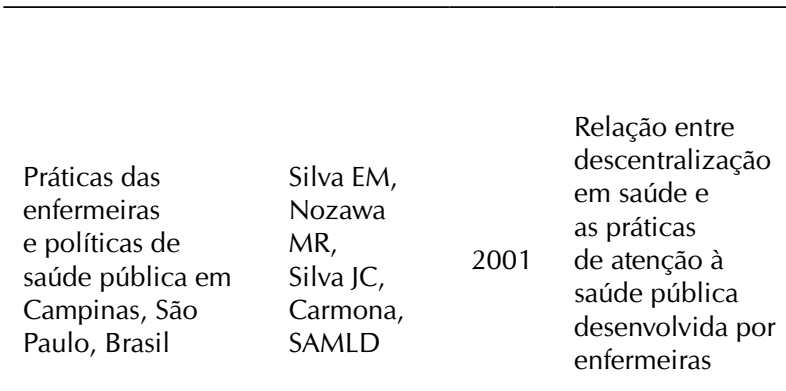

As práticas da enfermeira em saúde pública foram identificadas como burocráticas, educativas, e assistenciais, sendo que estas últimas são as mais enfatizadas.

Existe uma visão deformada ou preconceituosa das enfermeiras em relação à sua própria prática.

As enfermeiras, às vezes, executam atividades inerentes ao trabalho de outros profissionais.
A intervenção das enfermeiras tem se dirigido a diversas áreas, mas continua centrada nas consultas individuais, com pequena ênfase em atividades coletivas e de promoção à saúde.
Tendência das ações de educação em saúde realizadas por enfermeiros no Brasil
Lopes EM, Anjos SJSB, Pinheiro AKB
Práticas educativas em saúde implementadas por enfermeiros.
As ações de educação em saúde são voltadas tanto para pessoas sadias como para aquelas com doenças instaladas e em condições crônicas.
As ações de educação em saúde são efetivas para aquisição e compartilhamento de informações, possibilitando à clientela a execução de práticas favoráveis a sua saúde e seu bem-estar.

A participação da comunidade nas ações de educação em saúde é importante para a construção do autocuidado, da autonomia e das práticas de promoção da saúde.

Constatou-se que para a formação e condução de grupos há a necessidade de uma coordenação.

experiência sobre o processo educativo para a promoção da saúde de adolescentes
Relato de experiência sobre um

Hoga LAK, Abe CT 2000 trabalho desenvolvido com um grupo de adolescentes
A estratégia adotada possibilitou a exposição de dúvidas pelos adolescentes. É importante observar o objetivo da atividade educativa e o envolvimento das pessoas participantes do processo. 
A educação em saúde, assim como a promoção da saúde, é um campo multifacetado, para o qual convergem diversas concepções, as quais expressam diferentes ideologias e compreensões de saúde e de educação. Entre as várias tendências, duas destacam-se e persistem atualmente. Uma primeira, conhecida como tradicional, envolve a aprendizagem sobre doenças, como evitá-las, seus efeitos sobre a saúde e como restabelecê-la. A outra tendência inclui os fatores sociais que afetam a saúde, abordando os caminhos pelos quais diferentes estados de saúde e bem-estar são constituídos socialmente. Nesta segunda tendência, a aproximação entre os conceitos de educação em saúde e de promoção de saúde é muito evidente, havendo uma sobreposição entre eles. Entretanto, como já foi discutido nesse artigo, existem construções teóricas que apontam para diferenças significativas entre esses dois campos, sobretudo na amplitude e na especificidade das ações.

Compreende-se que a educação em saúde pode favorecer o aumento da autonomia das pessoas no seu cuidado e pode potencializar o exercício da cidadania, através do controle social sobre as políticas e serviços de saúde, contribuindo assim para a gestão social da saúde ${ }^{(17)}$ e para a construção e fortalecimento de ações no campo da promoção da saúde.

As duas vertentes da educação em saúde apontadas podem ser agrupadas em dois modelos: o modelo tradicional e o modelo dialógico ${ }^{(18)}$. A educação em saúde tradicional, também chamada de preventiva, é historicamente hegemônica, tendo como foco a doença e a intervenção curativa, e como embasamento teórico-prático o referencial biologicista do processo saúde-doença-cuidado. ${ }^{(19)}$ Baseado nos pressupostos da antiga saúde pública, o modelo tradicional da educação em saúde objetiva prevenir enfermidades e mudar comportamentos desfavoráveis à saúde. Seu enfoque é normativo e individual, baseando-se na ideologia comportamentalista, em que o indivíduo é o único responsável pelas suas condições de saúde ${ }^{(18)}$.

Nesse modelo, privilegiam-se as informações verticalizadas, que ditam comportamentos a serem adotados para a manutenção da saúde; os usuários são vistos como pessoas carentes de informação; a relação estabelecida entre profissionais e usuários é assimétrica, dado que os profissionais detêm um saber técnico-científico, com status de verdade, enquanto o outro precisa ser informado; e a relação profissional-usuário é marcada pelo caráter estritamente informativo, no qual o primeiro assume uma postura paternalista, explicitando hábitos e comportamentos saudáveis ${ }^{(19)}$.

O modelo dialógico da educação em saúde considera as raízes dos problemas de saúde e assume como objetivo central promover a saúde. Para o alcance desse objetivo na atenção primária, pressupõe-se a promoção de reflexões e a análise crítica sobre os aspectos da realidade pessoal e coletiva, visando desenvolver planos de transformação da realidade. Os pressupostos desse modelo são baseados nos constructos do educador Paulo Freire. Essa perspectiva auxilia tanto na análise coletiva dos problemas vivenciados pelos indivíduos como na busca de soluções conjuntas para a mudança da realidade ${ }^{(18)}$.

O objetivo da educação dialógica não é o de informar, mas de transformar os saberes existentes. A prática educativa, nesta perspectiva, visa desenvolver a responsabilidade dos indivíduos e da coletividade no cuidado com a saúde, não mais pela imposição de um saber técnico-científico restrito ao profissional de saúde, mas pelo desenvolvimento da compreensão da situação de saúde e das possibilidades de intervir sobre tal situação. Assim, a prática da educação em saúde mostra-se emancipatória e sensível às necessidades de saúde dos usuários ${ }^{(19)}$, constituindo uma estratégia fundamental para as enfermeiras promoverem a saúde na atenção primária.

Contudo, os quatro artigos que abordaram a educação em saúde enfatizaram-na como uma ferramenta para aconselhar, informar e mudar comportamentos e hábitos de vida, visando prevenir riscos à saúde. Dentre esses, dois artigos descreveram a educação em saúde com grupos de adolescentes, com vistas a mudar comportamentos e aconselhar o grupo sobre temas de interesse dos profissionais de saúde e do grupo. Um destes considerou a educação em saúde como estratégia para prevenir riscos à saúde de um grupo de tabagistas e outro a restringiu às orientações sobre a prática do aleitamento materno. Estes achados apontam para a presença, ainda marcante, da racionalidade preventivista no cotidiano de trabalho das enfermeiras que atuam na atenção primária, o que reduz a sua concepção de saúde à ausência de doenças.

O trabalho de grupo na atenção primária, voltado para a difusão de informações e aconselhamentos, objetivando mudanças de comportamentos, é importante para a prevenção de riscos e agravos à saúde. Porém, os profissionais de saúde que fornecem informações aos indivíduos ou a grupos tendem a acreditar que os sujeitos seguirão as orientações recebidas, ignorando suas crenças, valores e representações sobre o processo saúde-doença-cuidado e a possibilidade real destes seguirem as prescrições $^{(18)}$.

Sendo assim, a educação em saúde não pode ser concebida como um modo de impor à comunidade as compreensões e soluções consideradas corretas pelos profissionais, de maneira que as pessoas modifiquem os seus comportamentos considerados prejudiciais à saúde. É preciso compreender que a falta de higiene e de seguimento de muitas recomendações profissionais, por exemplo, ocorre por causas muito mais complexas do que a falta de informação e/ou a falta de motivação pessoal ${ }^{(20)}$, como o contexto socioeconômico e cultural onde o indivíduo está imerso.

Desenvolver trabalho de grupo na atenção primária é uma estratégia importante para o desenvolvimento da consciência crítica dos indivíduos a respeito do seu meio social e de suas condições de vida e saúde, o que possibilita o compartilhamento de conhecimentos que advém das experiências, além de potencializar processos coletivos para organizar e concretizar ações de mudança(18) ${ }^{(18}$ A educação em saúde deve ser realizada de modo que as pessoas possam identificar as causas primordiais de seus problemas, para poder encontrar as soluções a partir do diálogo/troca de saberes entre os profissionais de saúde e a própria comunidade ${ }^{(20)}$.

Contrapondo-se à perspectiva tradicional da educação em saúde abordada pelos quatro artigos mencionados, observou-se que os demais artigos sobre educação em saúde pautaram-se no modelo dialógico, evidenciando a preocupação em ampliar a abordagem do trabalho de grupo e a atuação da enfermeira na atenção primária. Foi consenso nesses artigos que as 
práticas educativas devem ir além de uma perspectiva preventiva, ampliando-se para uma práxis construtiva, pautada no desenvolvimento da cidadania e do diálogo. Estes aspectos, quando presentes na prática da enfermeira no contexto da APS, aproximam-se dos pressupostos da atenção primária e da promoção da saúde.

Um elemento comum a esses artigos foi a concepção de educação em saúde como uma estratégia que pode promover o vínculo e a horizontalização das relações entre profissionais e usuários na atenção primária à saúde, além de constituir uma ferramenta essencial para a troca de saberes entre os sujeitos, com vistas a romper a hegemonia do conhecimento técnico-científico nessa relação; estimular a participação popular e aumentar a autonomia dos sujeitos para que estes possam responsabilizar-se conjuntamente pela busca de soluções para os problemas individuais e coletivos e de novos meios para caminhar a vida.

Outros achados oriundos da análise desses artigos referem-se à preocupação com a construção de novas práticas na atenção primária, contrárias à abordagem puramente preventivista e biológica, convergindo para um modo ampliado de intervir no processo saúde-doença-cuidado e para a construção de práticas coletivas transformadoras. Além disso, dois estudos reconheceram a enfermeira como um agente capaz de atuar na melhoria das condições de vida e saúde da população, cuja profissão oferece subsídios para a construção de práticas em saúde pautadas no modelo dialógico da educação em saúde e na promoção da saúde.

Em um dos artigos chamou atenção a preocupação dos autores em conhecer as condições de vida e saúde das pessoas pertencentes ao grupo que eles estavam trabalhando. Isso pode ser percebido no momento em que eles relataram a preocupação em conhecer as relações sociais e os recursos disponíveis dos sujeitos, durante as visitas domiciliares, para a proposição de intervenções contextualizadas com a realidade das pessoas. Além disso, no transcorrer do relato, os autores reforçaram a necessidade de problematizar a realidade e explorar melhor os espaços tradicionais de atuação da enfermeira na atenção primária, como as visitas domiciliares e as consultas ambulatoriais, que na perspectiva deles, reveste-se de um caráter assistencial, educativo e dialógico.

Dentre os oito artigos que versaram sobre a educação em saúde, apenas um a reconheceu como uma estratégia a ser utilizada para o alcance da promoção da saúde. Os demais apenas citam a promoção da saúde, como se a educação em saúde e a promoção da saúde fossem sinônimos. Com isso, para procurar elementos que contribuíssem na compreensão da concepção de promoção da saúde que estava norteando os estudos, foi necessário analisar o referencial teórico que embasou os artigos. Nesse processo, observou-se que apenas um artigo utilizou os conceitos da promoção da saúde, limitando-se a defini-la sem estabelecer um limite entre ambas, o que demonstra que a promoção da saúde é muitas vezes tomada como educação em saúde e prevenção de doenças.

É preciso destacar que a educação em saúde deve ser compreendida como uma atividade-meio, ou seja, como uma estratégia que contribui para o alcance da promoção da saúde. As ações de promoção da saúde incluem a educação em saúde em seu campo de ação, mas vão além desta, pois visam provocar mudanças no âmbito organizacional, capazes de beneficiar as condições de vida e saúde da população de um modo geral(21).

Essa discussão reporta aos dois outros artigos incluídos nesse estudo. Um deles reitera a importância de se articular ações intersetoriais às práticas desenvolvidas por enfermeiras nas Unidades de Saúde da Família, como forma de responder adequadamente às complexas necessidades de saúde da população e como uma ferramenta para a promoção da saúde. Nesse estudo, ao analisar as concepções de enfermeiras sobre a intersetorialidade, concluiu-se que não há uma compreensão do que seria esta intersetorialidade, a qual é confundida com interdisciplinaridade. Esse fato faz com que seja necessário disseminar e aprofundar as discussões sobre este tema.

Um outro artigo, ao analisar as práticas da enfermeira no campo da saúde pública, concluiu que as intervenções da enfermeira nesse espaço continuam centradas nas consultas ambulatoriais, com pequena ênfase às atividades coletivas e de promoção da saúde. Estes achados demonstram que a prática da enfermeira na APS, muitas vezes, é orientada para a intervenção sobre o corpo individual, reiterando a lógica de uma prática individual, prescritiva e focada na doença ${ }^{(22)}$.

\section{CONCLUSÕES}

Este estudo, ao analisar a produção científica nacional sobre a promoção da saúde na prática da enfermeira no campo da atenção primária, identificou que a promoção da saúde é pouco revelada na prática de enfermeiras que atuam nesse âmbito da atenção à saúde. O baixo porcentual (4\%) de artigos incluídos no estudo sinaliza que a produção de conhecimento pelas enfermeiras sobre a promoção da saúde na atenção primária é incipiente e pouco explorada. Além disso, depreende-se que a promoção da saúde ainda é tida como sinônimo da educação em saúde, particularmente no modelo tradicional, fundamentado em ações prescritivas de prevenção de doenças.

A situação acima reforça a necessidade de ampliar a compreensão da educação em saúde, entendendo-a como um campo de práticas e de conhecimento que tem se ocupado mais com a criação de vínculos entre os serviços de saúde e o modo de pensar e agir cotidiano da população. Como prática transversal, a educação em saúde proporciona a articulação entre todos os níveis de gestão do sistema, representando um dispositivo essencial tanto para formulação de políticas de saúde de forma compartilhada, como para a integração entre saberes e práticas que se coloquem a favor da vida, da dignidade, do respeito ao outro, da humanização e da integralidade das ações de saúde. É nesse sentido que se pode pensar na educação em saúde, como uma estratégia fundamental para o alcance da promoção da saúde no processo saúde-doença-cuidado.

Não se pode deixar de destacar que os profissionais de saúde, e dentre eles as enfermeiras, são desafiados a todo o momento em sua prática cotidiana nos serviços de atenção primária. Isso acontece principalmente devido aos inúmeros problemas da gestão do sistema, da medicalização da saúde, da incipiência da educação permanente, da hegemonia do paradigma biomédico 
na sociedade e falta de compreensão por parte dos profissionais de saúde quanto à amplitude da sua prática.

A permanência desta situação contribui para a falta de reconhecimento da promoção da saúde como uma estratégia que pode impulsionar transformações na prática das enfermeiras e dos demais profissionais que atuam na atenção primária, e produzir repercussões importantes sobre o estado de saúde da população.

Considerando que a promoção da saúde é capaz de responder aos complexos problemas inerentes a atenção à saúde da população, é crucial nesse momento que as enfermeiras que atuam na atenção primária comecem a compreender a promoção da saúde como um campo de práxis capaz de transformar a realidade social e a sua própria prática.

Não se pretende com este estudo atingir objetivos conclusivos. A discussão aqui realizada e os achados oriundos da aproximação entre a promoção da saúde e a prática da enfermeira na atenção primária fornecem subsídios para estudos posteriores sobre as diversas nuances dessa temática. Dentre elas vale aprofundar o que explica a incompreensão das enfermeiras sobre o conceito de promoção da saúde, confundida com educação em saúde; sobre a incipiência das ações de promoção da saúde na prática da enfermeira na APS; sobre o próprio conteúdo das ações da enfermeira na APS e sobre o desinteresse que este campo de investigação tem para as produtoras do conhecimento no campo da enfermagem.

\section{REFERÊNCIAS}

1. Heidmann ITSB, Almeida MCP, Boehs AE, Wosny AM, Monticelli M. Promoção à saúde: trajetória histórica de suas concepções. Texto Contexto \& Enferm 2006;15(2):352-8.

2. Sícoli JL, Nascimento PR. Promoção de saúde: concepções, princípios e Operacionalização. Interface Comunic Saúde Educ 2003;7(12):101-22.

3. Czeresnia D. O conceito de saúde e a diferença entre prevenção e promoção. In: Czeresnia D, Freitas CM, organizadores. Promoção da saúde: conceitos, reflexões, tendências. Rio de Janeiro: Fiocruz; 2009. p. 39-54.

4. Buss PM. Uma introdução ao conceito de promoção da saúde. In: Czeresnia D, Freitas CM, organizadores. Promoção da saúde: conceitos, reflexões, tendências. Rio de Janeiro: Fiocruz; 2009. p. 15-38.

5. Arouca S. O dilema preventivista: contribuição para a compreensão e crítica da Medicina Preventiva. São Paulo: Unesp; Rio de Janeiro: Ed. Fiocruz; 2003.

6. Westphal MF. Promoção da saúde e prevenção de doenças. In: Campos GWS, Minayo MCS, Akerman M, Drumond Jr M, Carvalho YMC, Organizadores. Tratado de Saúde Coletiva. Rio de Janeiro: Fiocruz; 2009. p. 635-67.

7. Mascarenhas NB. Promoção da saúde e a prática do enfermeiro na atenção primária: contribuição ao estudo. Salvador. Monografia [Graduação em Enfermagem]- Escola de Enfermagem, Universidade Federal da Bahia; 2010.

8. Starfield B. Atenção Primária: equilíbrio entre necessidades de saúde, serviços e tecnologias. Brasília: UNESCO/ Ministério da Saúde; 2002.

9. Andrade LOM, Barreto ICHC, Bezerra RC. Atenção Primária à Saúde e Estratégia de Saúde da Família. In: Campos GWS, Minayo MCS, Akerman M, Drumond Jr M, CarvaIho YMC, Organizadores. Tratado de Saúde Coletiva. Rio de Janeiro: Fiocruz; 2009. p. 783-836.

10. Silva KL, Sena RR, Grillo MJC, Horta NC, Prado PMC. Educação em enfermagem e os desafios para a promoção de saúde. Rev Bras Enferm 2009;62(1):86-91.
11. Collière M-F. Cuidar... A primeira arte da vida. 2. ed. Loures: Lusociência; 2003.

12. Collière M-F. Promover a vida. 3. Ed. Lisboa: Lidel e Sindicato dos Enfermeiros Portugueses; 1999.

13. Mendes KDS, Silveira RCCP, Galvão CM. Revisão integrativa: método de pesquisa para a incorporação de evidências na saúde e na enfermagem. Texto Contexto \& Enferm 2008; 17(4):758-64.

14. Sampaio RF, Mancini MC. Estudos de revisão sistemática: um guia para síntese criteriosa da evidência científica. Rev Bras Fisioter 2007; 11(1):83-9.

15. Minayo MCS, Deslandes SF, Gomes R. Pesquisa Social: teoria, método e criatividade. Rio de Janeiro: Editora Petrópolis; 2007.

16. Lira SVG, Bezerra MPB, Frota MA, Valdés MTM, Vieira LJES, Silva RM. Produção científica sobre promoção da saúde nos cursos de pós-graduação brasileiros. Saúde Soc 2009;18(3):437-45.

17. Ministério da Saúde. Conselho Nacional das Secretarias Municipais de Saúde. SUS de A a Z: garantindo saúde nos municípios. Brasília; 2009. (Série F. Comunicação e Educação em Saúde).

18. Souza AC, Colomé ICS, Costa LED, Oliveira DLLC. A educação em saúde com grupos na comunidade: uma estratégia facilitadora da promoção da saúde. Rev Gaúch Enferm 2005;26(2): 147-53.

19. Alves VS. Um modelo de educação em saúde para o Programa de Saúde da Família: pela integralidade da atenção e reorientação do modelo assistencial. Interface Comunic Saúde Educ 2005;9(16):39-52.

20. Vasconcelos EM. Educação popular nos serviços de saúde. São Paulo: Editora Hucitec; 1997.

21. Candeias NMF. Conceitos de educação e de promoção em saúde: mudanças individuais e mudanças organizacionais. Rev Saúde Pública 1997;31(2):209-13.

22. Ermel RC, Fracolli LA. O trabalho das enfermeiras no Programa de Saúde da Família em Marília/SP. Rev Esc Enferm USP 2006;40(4):533-9. 